\title{
Ionic to neutral conversion induced by resonant excitation of molecular vibrations coupled to intermolecular charge transfer
}

\author{
T. Morimoto, ${ }^{1}$ H. Suzuki, ${ }^{1}$ T. Otaki, ${ }^{1}$ N. Sono,${ }^{1}$ N. Kida,,${ }^{1,2}$ T. Miyamoto,${ }^{1,2, *}$ and H. Okamoto $\odot^{1,2, \dagger}$ \\ ${ }^{1}$ Department of Advanced Materials Science, University of Tokyo, Kashiwa 5-1-5, Chiba 277-8561, Japan \\ ${ }^{2}$ AIST-UTokyo Advanced Operando-Measurement Technology Open Innovation Laboratory, \\ National Institute of Advanced Industrial Science and Technology, Chiba 277-8568, Japan
}

(Received 23 March 2020; revised 16 August 2021; accepted 22 October 2021; published 18 November 2021)

\begin{abstract}
In organic molecular compounds, intramolecular vibration is sometimes coupled with intermolecular charge transfer (CT). In such materials, vibrational excitation by a midinfrared (MIR) pulse causes collective intermolecular CTs that can be a route to an electronic-state conversion. Here, we report that an ionic-to-neutral (IN) conversion in tetrathiafulvalene-p-chloranil (TTF-CA) can be driven by a strong vibrational excitation induced by an MIR pulse. Using MIR-pump subcycle-reflectivity-probe and second-harmonic-generation-probe measurements, we discuss the coherent electron and lattice dynamics during and after the IN conversion, which are distinct from the dynamics of the photoinduced transition by electronic excitation alone.
\end{abstract}

DOI: 10.1103/PhysRevResearch.3.L042028

A solid irradiated with a femtosecond laser pulse sometimes shows a drastic change in its electronic or crystal structure, which is called a photoinduced phase transition (PIPT) [1]. In most PIPTs, the excitation of an interband transition by a visible or near-infrared (NIR) pulse generates photocarriers, which give rise to a phase transition via cooperative electron-electron and electron-lattice interactions. Recent developments in femtosecond laser techniques enable us to generate tunable far-IR and mid-IR (MIR) pulses with frequencies of $0.5-100 \mathrm{THz}$. Such a pulse can excite not only an electronic transition but also a phonon and magnon. Resonant excitation of specific electronic transition or fundamental mode is attracting attention as a way to drive PIPTs. Examples include the control of magnetization or spin state via the excitation of magnons, electro-magnons, and phonons in magnetic materials [2-6]; the control of superconductivity via phonon [7-9] or electronic excitations [10]; the control of intersite orbital overlap in correlated oxides [11]; and higher-order phonon generation and polarization control via the phonon excitation in dielectrics [12-16].

Here, we focus on the resonant excitation of intramolecular vibrations in organic charge transfer (CT) compounds with an intense MIR pulse. Previous studies reported that such a vibrational excitation in bis(ethylenedithio)tetrathiafulvalene (ET)-based CT compounds modified an electronic state via a modulation of an onsite Coulomb repulsion of ET $[9,17,18]$, while an electronic-state conversion has not

\footnotetext{
*miyamoto@k.u-tokyo.ac.jp

†okamotoh@k.u-tokyo.ac.jp
}

Published by the American Physical Society under the terms of the Creative Commons Attribution 4.0 International license. Further distribution of this work must maintain attribution to the author(s) and the published article's title, journal citation, and DOI. been achieved. In CT compounds, intramolecular vibrations are sometimes coupled with intermolecular CTs via electron intramolecular vibration (EMV) coupling $[19,20]$. Therefore, the strong excitation of an intramolecular vibration could effectively induce intermolecular CTs, which could be a route to an electronic-state conversion. In this Letter, we report that an ionic-to-neutral (IN) conversion can be induced in tetrathiafulvalene- $p$-chloranil (TTFCA) by exciting intramolecular vibrations using a carrierenvelope-phase (CEP)-stable MIR pulse. With the results of MIR-pump subcycle-reflectivity-probe and second-harmonicgeneration (SHG)-probe measurements, we discuss the electron and lattice dynamics involved in this phonon-driven IN conversion.

In TTF-CA, donor $(D)$ molecules of TTF and acceptor $(A)$ molecules of CA [Fig. 1(a)] arrange alternately along the $a$ axis, forming a quasi-one-dimensional (1D) electronic state [21-23]. This electronic state is characterized by the degree of CT from $A$ to $D$ molecules $\rho$ [21]. At room temperature, TTF-CA is a van der Waals neutral crystal with $\rho_{\mathrm{N}} \sim 0.3$ [Fig. 1(b)]. It undergoes an neutral-to-ionic transition (NIT) at $T_{\mathrm{c}}=81 \mathrm{~K}$, below which it is an ionic crystal with $\rho_{\mathrm{I}} \sim$ 0.6 [Fig. 1(c)] [22,24]. The NIT mechanism is explained as follows. With decrease in temperature, the intermolecular distance along the 1D molecular stacks decreases. This enhances the energy gain by long-range Coulomb attractive interactions between $D$ and $A$ molecules, which overcomes the ionization energy of $D A$ pairs. In I phase, $D$ and $A$ molecules are dimerized because of the spin-Peierls mechanism. The order parameter of this NIT is the dimerization [25]. The important feature of the NIT is that a ferroelectric polarization appears in I phase, which originates from collective intermolecular CTs $(\delta \rho \sim 0.2)$ at NIT [the curved arrows in Fig. 1(c)] [26-28]. Thus, TTF-CA is an electronic-type ferroelectric. Since the electronic component of the polarization is opposite to the direction of the ionic one, and the former is much larger 


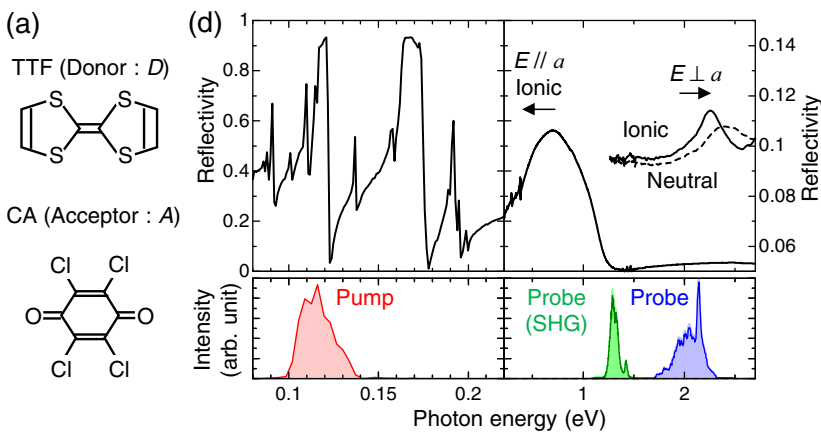

(b) Neutral phase

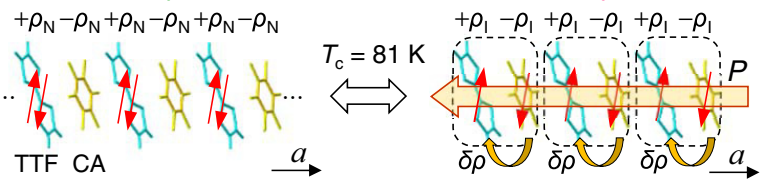

(e)

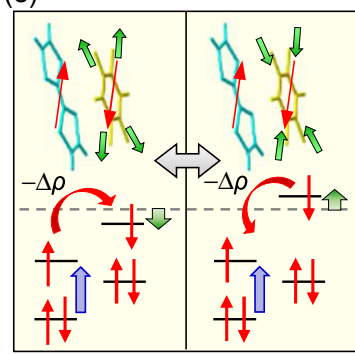

(f)

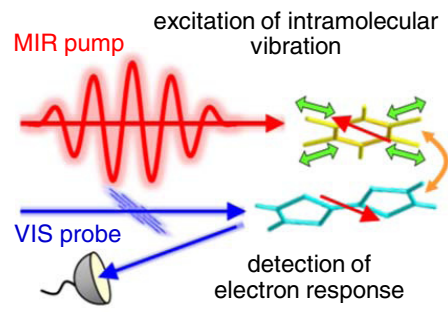

FIG. 1. (a) Molecular structures of tetrathiafulvalene (TTF) and chloranil (CA). (b) and (c) One-dimensional (1D) molecular stacks in neutral and ionic phases. $P$ is the ferroelectric polarization. (d) Polarized reflectivity spectra of TTF-CA at $77 \mathrm{~K}$ (ionic phase) and $90 \mathrm{~K}$ (neutral phase). The red line shows a spectrum of pump pulses. The blue (green) line shows a spectrum of probe pulses used in the reflectivity [second harmonic generation (SHG)] measurement. (e) A schematic of an electron intramolecular vibration (EMV) coupling. The red arrows show electron transfers synchronized with the $a_{g}$ mode oscillation of CA. (f) A schematic of the midinfrared (MIR)pump visible-probe measurement.

than the latter, the dimerization is not a driving force of the ferroelectric transition [26,29,30].

Figure 1(d) shows the polarized reflectivity $(R)$ spectra of TTF-CA in I phase $(77 \mathrm{~K})$, measured with the electric field of the light $(E)$ parallel $(/ /)$ and perpendicular $(\perp)$ to the $a$ axis. The structure $\sim 0.6 \mathrm{eV}$ for $E / / a$ is the CT band between $D$ and $A$ molecules. The sharp structures $<0.2 \mathrm{eV}$ are ascribed to totally symmetric $\left(a_{g}\right)$ modes of intramolecular vibrations activated by dimerization [24,31,32]. The band $\sim 2.3 \mathrm{eV}$ for $E \perp a$ is assigned to the intramolecular transition from the highest-occupied molecular orbital (MO) to the singly occupied MO in TTF cations [the blue arrows in Fig. 1(e)] [33,34]. This band shifts to a lower energy and increases in intensity as $\rho$ increases [33-36], so it is a good probe for NIT.

The MIR-pump visible-probe experiment is illustrated in Fig. 1(f). A CEP-stable MIR pulse is obtained using a previously reported method [37]. The typical electric field waveform $E_{\mathrm{MIR}}(t)$ is shown with a red line in Fig. 2(a). Its Fourier power spectrum is shown with the red lines in Figs. 1(d) and 2(c). In Fig. 2(c), we also show the $\varepsilon_{2}$ spectrum
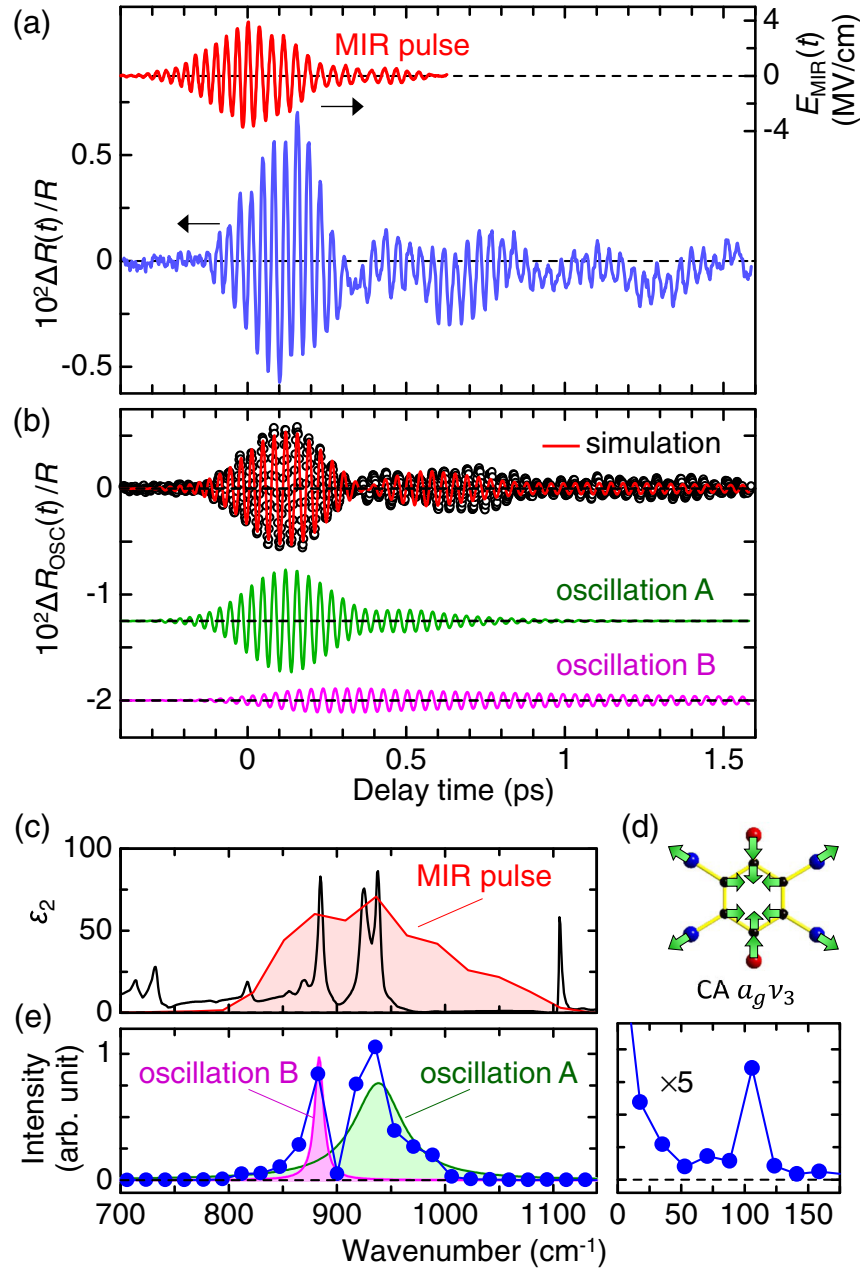

FIG. 2. (a) Time evolution of $\Delta R(t) / R$ of the visible probe pulse (the blue line) at $50 \mathrm{~K}$ by the midinfrared (MIR) electric field pulse (the red line). (b) High-frequency oscillatory component $\Delta R_{\mathrm{OSC}}(t) / R$ (open circles). The red line shows a fitting curve consisting of oscillations A and B presented in the lower part. (c) The spectra of the MIR pulse and $\varepsilon_{2}$ for $E / / a(77 \mathrm{~K})$. (d) Schematic of $a_{g} \nu_{3}$ mode of CA. (e) Fourier power spectrum of $\Delta R(t) / R$ (blue circles) and those obtained from the analysis of $\Delta R_{\mathrm{OSC}}(t) / R$.

along the $a$ axis. The MIR pulse excites three vibrational modes $\sim 900 \mathrm{~cm}^{-1}$. The peak at $940 \mathrm{~cm}^{-1}$ was assigned to the $a_{g} v_{3}$ mode of CA, which is mainly composed of the in-phase combination of four $\mathrm{C}-\mathrm{Cl}$ stretching vibrations [Fig. 2(d)] [32]. This $a_{g}$ mode is IR inactive in an isolated molecule. As illustrated in Fig. 1(e), the $a_{g}$ mode can modulate the MO energy via EMV coupling to induce a partial $\mathrm{CT}$ in a $D A$ dimer $\pm \Delta \rho$. In $\mathrm{N}$ phase with inversion symmetry, this mode is still IR inactive, while in I phase with dimerization, the mode becomes IR active. Two peaks at 925 and $885 \mathrm{~cm}^{-1}$ are assigned to the modes of CA and TTF, respectively, which are activated by similar EMV couplings [32]. The visibleprobe pulse is generated by a noncollinear optical parametric amplifier [38,39]. Its temporal width (10.5 fs) is much shorter than the period of the MIR electric field, $\sim 35 \mathrm{fs}$, which allows subcycle measurement. The central energy of this pulse is 2.05 $\mathrm{eV}$ [the blue line in Fig. 1(d)]. The $R$ value for this pulse $(E \perp a)$ decreases as $\rho$ decreases and therefore can be used to 


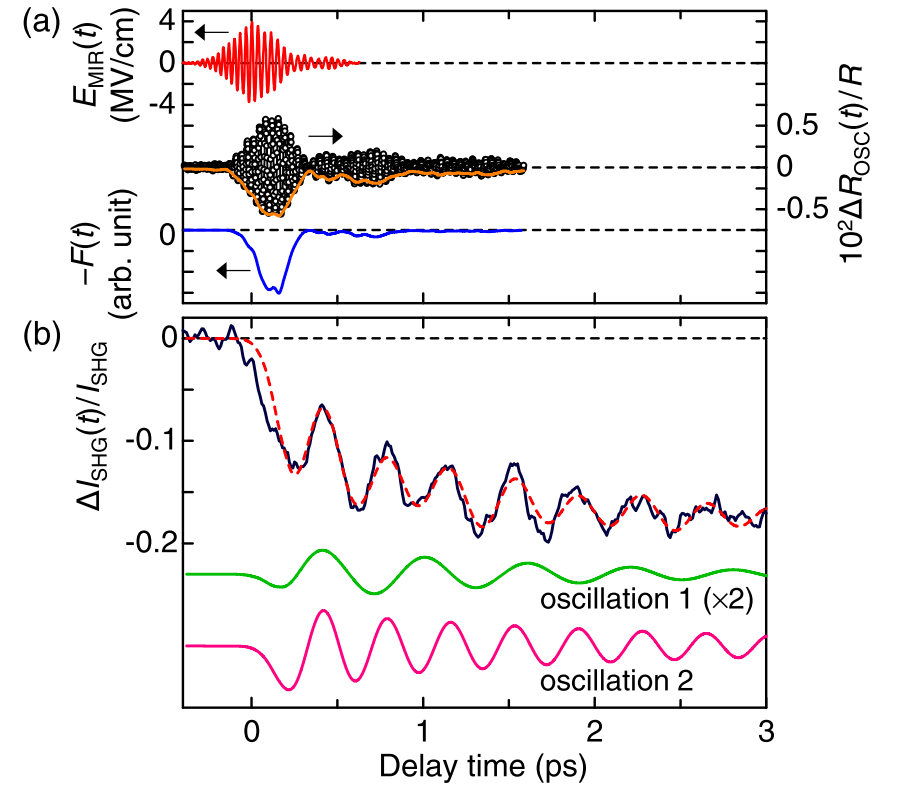

FIG. 3. (a) $E_{\mathrm{MIR}}(t)$ and $\Delta R_{\mathrm{OSC}}(t) / R$ shown in Figs. 2(a) and 2(b). The orange and blue lines show the envelope of $\Delta R_{\mathrm{OSC}}(t) / R$ and its square $F(t)$, respectively. (b) $\Delta I_{\mathrm{SHG}}(t) / I_{\mathrm{SHG}}$ (the black line) and the fitting curve (the red broken line). Two oscillatory components are shown in the lower part.

probe INT. For the MIR-pump SHG-probe measurements, an incident probe pulse $(E / / a)$ with photon energy of $1.3 \mathrm{eV}$ and temporal width of $\sim 25 \mathrm{fs}$ was used. The long temporal width does not allow subcycle measurements, so the change in the SHG intensity $I_{\text {SHG }}$ averaged within each pulse was detected. The experimental details are reported in Supplemental Material S1 [40].

The blue line in Fig. 2(a) shows the time evolution of reflectivity changes $\Delta R(t) / R$ in TTF-CA $(50 \mathrm{~K})$ by an MIR pulse with $E_{\mathrm{MIR}} / / a$. The maximum electric field amplitude $E_{\mathrm{MAX}}$ is $4.0 \mathrm{MV} / \mathrm{cm}$. Here, $R(t)$ is modulated rapidly within a period of $\sim 35$ fs. In Fig. 2(e), we show the Fourier power spectra of $\Delta R(t) / R$ (blue circles). In the high-frequency region, the spectrum features two peaks, which correspond to those of the $\varepsilon_{2}$ spectrum, although the doublet $\sim 930 \mathrm{~cm}^{-1}$ is not resolved. We also performed the fitting analyses of the high-frequency oscillatory component $\Delta R_{\mathrm{OSC}}(t) / R$ [the open circles in Fig. 2(b)] (Supplementary Material S2 [40]). By assuming two damped oscillators $\mathrm{A}$ and $\mathrm{B}, \Delta R_{\mathrm{OSC}}(t) / R$ is well reproduced, as shown by the red line in Fig. 2(b). The time profile and Fourier power spectrum of each oscillation are shown in Figs. 2(b) and 2(e), respectively. The frequency is $939 \mathrm{~cm}^{-1}\left(883 \mathrm{~cm}^{-1}\right)$ for oscillation A (B). These oscillations are ascribed to the modulation of $\rho, \pm \Delta \rho$, by $a_{g}$ mode vibrations. Notably, oscillation A originates from the $a_{g}$ mode of CA, while the modulations of $\rho$ in TTF are detected as reflectivity changes. This is unambiguous evidence of collective intermolecular CT via EMV coupling. The Fourier power spectrum in the low-frequency region shows a peak $\sim 100 \mathrm{~cm}^{-1}$, although its frequency cannot be determined because of the coarse spectral resolution.

As seen in Fig. 2(a), $\Delta R(t) / R$ for $t>0.2 \mathrm{ps}$ is negative on average, which shows a decrease in the averaged $\rho$ that is

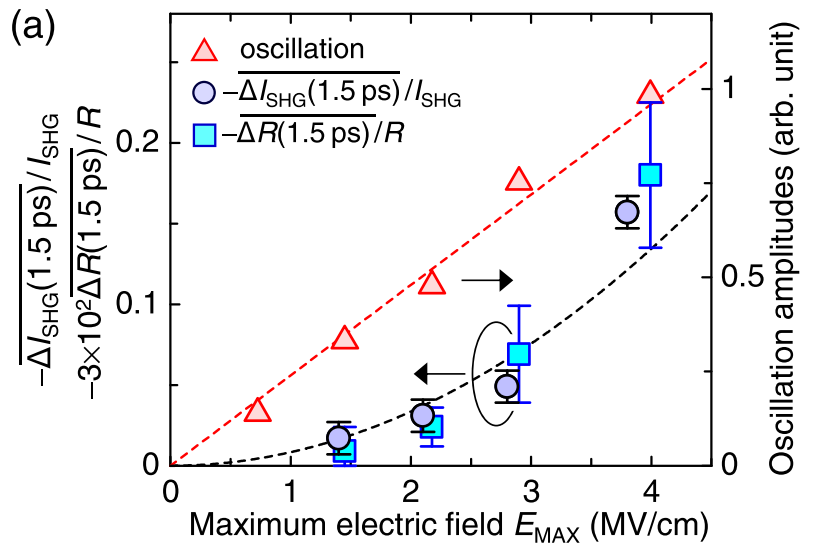

(b)

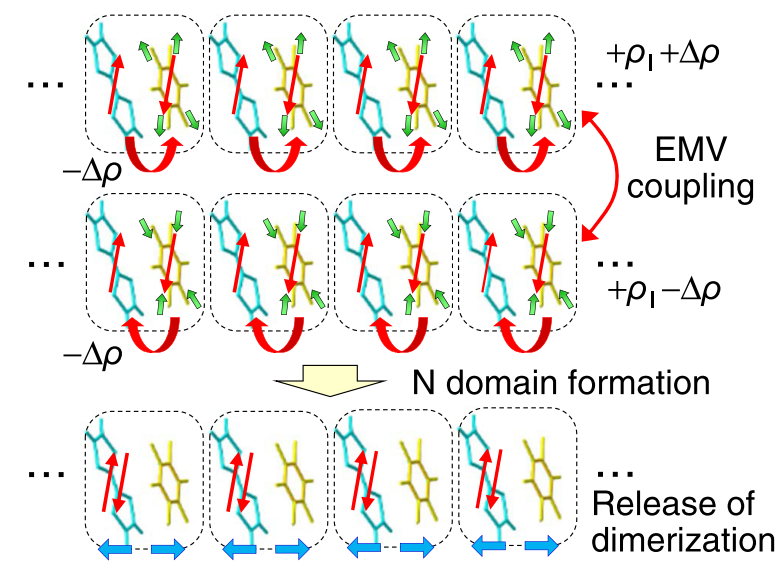

FIG. 4. (a) Magnitudes of $-\overline{\Delta R(1.5 \mathrm{ps})} / R$ and $-\overline{\Delta I_{\mathrm{SHG}}(1.5 \mathrm{ps})} / I_{\mathrm{SHG}}$ reflecting the ionic-to-neutral (IN) conversion efficiency and amplitudes of the high-frequency oscillation as a function of $E_{\mathrm{MAX}}$. (b) Schematic of IN conversion dynamics induced by the midinfrared (MIR) pulse.

reminiscent of INT. To investigate this phenomenon, we performed MIR-pump SHG-probe measurements at $50 \mathrm{~K}$. Since SHG occurs only in I phase, the decrease in SHG intensity $I_{\text {SHG }}$ can serve as a measure of the efficiency of INT [43]. In Fig. 3(b), we show the change in $I_{\mathrm{SHG}}, \Delta I_{\mathrm{SHG}}(t) / I_{\mathrm{SHG}}$, by the same MIR pulse as that used for measuring $\Delta R(t) / R$ in Fig. 2(a). Here, $I_{\mathrm{SHG}}$ shows a stepwise decrease up to $\sim 0.15 \mathrm{ps}$, a subsequent gradual decrease up to $1.5 \mathrm{ps}$, and a slow oscillation, which is also shown in $\Delta R(t) / R$ [Fig. 2(a)]. From the magnitude of $-\Delta I_{\mathrm{SHG}}(t) / I_{\mathrm{SHG}}$ at $3 \mathrm{ps}, \sim 20 \%$, the decrease of the original polarization $P, \Delta P / P$, is estimated to be $\sim 10 \%[29,44]$. This decrease suggests that $\sim 10 \%$ of the ionic state is converted to the neutral state. The time characteristics of $\Delta I_{\mathrm{SHG}}(t) / I_{\mathrm{SHG}}$ will be discussed later.

To clarify the IN conversion mechanism, we investigated how the magnitudes of $\Delta R(t) / R$ and $\Delta I_{\mathrm{SHG}}(t) / I_{\mathrm{SHG}}$ signals excepting the oscillatory components, $\overline{\Delta R(t)} / R$ and

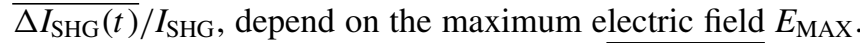
Figure 4(a) plots these values at $1.5 \mathrm{ps},-\overline{\Delta R(1.5 \mathrm{ps})} / R$ and $-\overline{\Delta I_{\mathrm{SHG}}(1.5 \mathrm{ps})} / I_{\mathrm{SHG}}$, as a function of $E_{\mathrm{MAX}}$ together with the relative amplitude of the high-frequency oscillations, $A_{\mathrm{OSC}}$. The latter is obtained by taking the square root of the integral of the Fourier power spectrum (blue circles) in the left panel of Fig. 2(e). Here, $A_{\mathrm{OSC}}$ is proportional to $E_{\mathrm{MAX}}$, while 
$\overline{\Delta R(1.5 \mathrm{ps})} / R$ and $\overline{\Delta I_{\mathrm{SHG}}(1.5 \mathrm{ps})} / I_{\mathrm{SHG}}$ are proportional to the square of $E_{\mathrm{MAX}}$, at least for $E_{\mathrm{MAX}}<3 \mathrm{MV} / \mathrm{cm}$. This means that the efficiency of IN conversion is proportional to the square of the intramolecular vibration amplitude.

Considering these results, we discuss the mechanism of IN conversion induced by the MIR pulse. Here, $\Delta I_{\text {SHG }}(1.5 \mathrm{ps}) / I_{\text {SHG }}$ does not show a threshold behavior, indicating that no electric-field-induced tunneling processes are involved. As seen in Fig. 2(a), the resonant excitation of intramolecular vibrations gives rise to the modulation of $\rho( \pm \Delta \rho)$. From the maximum (minimum) value of $\Delta R_{\mathrm{OSC}}(t) / R$, this modulation is estimated to be $\sim \pm 0.074$ at $E_{\mathrm{MAX}}=4 \mathrm{MV} / \mathrm{cm}$ (Supplemental Material S3 [40]). This is $\sim \pm 40 \%$ of the change in $\rho(\delta \rho \sim 0.2$ [24] $)$ at the temperature-induced NIT. The modulation of $\rho$ by the MIR electric field causes the mixing of wave functions of ionic and neutral states. In a simple dimer model, the wave function of the ground state $\left|\psi_{\mathrm{GS}}\right\rangle$ is expressed as follows when it is modified by an EMV coupling [31]:

$$
\left|\psi_{\mathrm{GS}}\right\rangle \propto\left|\psi_{\mathrm{I}}\right\rangle+\frac{\sqrt{\rho(1-\rho)}}{\omega_{\mathrm{CT}}} \tilde{\omega}_{m}^{2} I_{m} Q_{m}\left|\psi_{\mathrm{N}}\right\rangle
$$

Here, $\left|\psi_{\mathrm{I}}\right\rangle$ and $\left|\psi_{\mathrm{N}}\right\rangle$ are the wave functions of the ionic ground state and the neutral excited state, respectively. Also, $\omega_{\mathrm{CT}}$ and $\tilde{\omega}_{m}$ are the frequency of the CT transition and the intramolecular vibration, respectively. Further, $I_{m}$ is a parameter proportional to the EMV coupling constant. Then $Q_{m}$ is a normal coordinate of the intramolecular vibration. Equation (1) shows that the neutral excited state is mixed with the ionic ground state via intramolecular vibration. Using Eq. (1), we obtain the expectation value of $\rho,\left\langle\psi_{\mathrm{GS}}|\hat{\rho}| \psi_{\mathrm{GS}}\right\rangle$, as follows:

$$
\begin{aligned}
\left\langle\psi_{\mathrm{GS}}|\hat{\rho}| \psi_{\mathrm{GS}}\right\rangle= & \left|c_{1}(t)\right|^{2} \rho_{\mathrm{I}}+\left|c_{2}(t)\right|^{2} \rho_{\mathrm{N}} \\
& +c_{1}^{*}(t) c_{2}(t)\left\langle\psi_{\mathrm{I}}|\hat{\rho}| \psi_{\mathrm{N}}\right\rangle \\
& +c_{1}(t) c_{2}^{*}(t)\left\langle\psi_{\mathrm{N}}|\hat{\rho}| \psi_{\mathrm{I}}\right\rangle .
\end{aligned}
$$

Here, $\quad\left|\psi_{\mathrm{GS}}\right\rangle=c_{1}(t)\left|\psi_{\mathrm{I}}\right\rangle+c_{2}(t)\left|\psi_{\mathrm{N}}\right\rangle, \quad \rho_{\mathrm{I}}=\left\langle\psi_{\mathrm{I}}|\hat{\rho}| \psi_{\mathrm{I}}\right\rangle$, and $\rho_{\mathrm{N}}=\left\langle\psi_{\mathrm{N}}|\hat{\rho}| \psi_{\mathrm{N}}\right\rangle$. Taking the time average of Eq. (2), the third and fourth terms disappear, and the average value of $\rho$ decreases $\left(\left\langle\psi_{\mathrm{GS}}|\hat{\rho}| \psi_{\mathrm{GS}}\right\rangle<\rho_{\mathrm{I}}\right)$ via the second term $\left|c_{2}(t)\right|^{2} \rho_{N}$. Equation (1) indicates $c_{2}(t) \propto Q_{m}$, which should be proportional to $E_{\mathrm{MAX}}$. As a result, the decrease in the average value of $\rho$ is proportional to $\left(E_{\mathrm{MAX}}\right)^{2}$. This is consistent with the experimental result that $-\overline{\Delta R(1.5 \mathrm{ps})} / R$ and $-\overline{\Delta I_{\mathrm{SHG}}(1.5 \mathrm{ps})} / I_{\mathrm{SHG}}$ are proportional to $\left(E_{\mathrm{MAX}}\right)^{2}$ [Fig. 4(a)]. The amplitude of the charge modulation reaches the maximum at $t \sim 0.13 \mathrm{ps}$ at which the energy given by the MIR pulse is stored in this vibration. At this time $(t \sim 0.13 \mathrm{ps})$, the decrease in the SHG intensity $\left|\Delta I_{\mathrm{SHG}}(t) / I_{\mathrm{SHG}}\right| \sim 0.1$ already reached $60 \%$ of its total decrease of $\left|\Delta I_{\mathrm{SHG}}(t) / I_{\mathrm{SHG}}\right| \sim 0.17$. Such a rapid decrease of the SHG intensity occurring before the oscillation decays indicates that the neutral-domain formation is not caused by the heating of the system (see Supplemental Material S4 for details [40]). Eqs. (1) and (2) predict the mixing of wave functions of neutral and ionic states by the MIR electric field, while the experimental result shows that the decrease in the average $\rho$ persists at least up to $3 \mathrm{ps}$. This suggests that the mixing of the neutral state with the ionic state gives rise to the formation of stable neutral domains [Fig. 4(b)]. According to previous NIR-pump visible-reflectivity-probe studies, neutral domains composed of $\sim 10-20 D A$ pairs are indeed generated from a CT-excited state in the ionic phase and have lifetimes $>100 \mathrm{ps}$ [34].

We next analyze the time evolution of $\Delta I_{\mathrm{SHG}}(t) / I_{\mathrm{SHG}}$ [Fig. 3(b)]. When an MIR pulse generates a high-frequency oscillation of $\rho$ and $\rho$ approaches that of the neutral phase, a neutral domain is generated, the probability of which is expressed by $F(t)$. As discussed above, $F(t)$ is proportional to the square of the charge-modulation amplitude $(\Delta \rho)^{2}$ induced by the MIR electric field. Here, $\Delta R_{\mathrm{OSC}}(t) / R$ is proportional to $\Delta \rho$, so that $F(t)$ should be proportional to the square of the envelope profile of $\Delta R_{\mathrm{OSC}}(t) / R$. Using the Hilbert transform, we calculate the envelope profile of $\Delta R_{\mathrm{OSC}}(t) / R$ [the orange line in Fig. 3(a)] and obtain $F(t)$ as shown by the blue line in Fig. 3(a). The initial rapid decrease of $I_{\mathrm{SHG}}$ at $\sim 0.1 \mathrm{ps}$, reflecting the formation of neutral domains, can be expressed by a step function convoluted with $F(t)$. Based on these discussions, we analyze $\Delta I_{\mathrm{SHG}}(t) / I_{\mathrm{SHG}}$ using the following formula:

$$
\begin{aligned}
\frac{\Delta I_{\mathrm{SHG}}(t)}{I_{\mathrm{SHG}}} \propto & \int_{-\infty}^{\infty} F(\tau) g(t-\tau) d \tau, \\
g(t)= & \left\{C_{1}+C_{2}\left[1-\exp \left(-\frac{t}{\tau_{r}}\right)\right]\right. \\
& \left.+\sum_{i=1}^{2}\left[D_{i} \exp \left(-\frac{t}{\tau_{i}}\right) \cos \left(\omega_{i} t+\varphi_{i}\right)\right]\right\} \theta(t) .
\end{aligned}
$$

Here, $\Delta I_{\mathrm{SHG}}(t) / I_{\mathrm{SHG}}$ is expressed by the function $g(t)$ convoluted with $F(t)$. Also, $\theta(t)=0$ for $t<0$ and $\theta(t)=1$ for $t \geqslant 0$. In $g(t)$, the first term $C_{1} \theta(t)$ expresses the initial neutral-domain formation. The second term shows the subsequent slow decrease of $\Delta I_{\mathrm{SHG}}(t) / I_{\mathrm{SHG}}$ with the characteristic time $\tau_{r}$. The third term expresses the coherent oscillations. We take two damped oscillators into account. Using Eq. (3), the time evolution of $\Delta I_{\mathrm{SHG}}(t) / I_{\mathrm{SHG}}$ is well reproduced, as shown by the broken line in Fig. 3(b). Mode 1 (2) has the frequency $\omega_{1} \sim 56 \mathrm{~cm}^{-1}\left(\omega_{2} \sim 89 \mathrm{~cm}^{-1}\right)$, and its time evolution is expressed by $-\cos \left(\omega_{1} t\right)\left[-\sin \left(\omega_{2} t\right)\right]$. Those oscillatory components are also shown in Fig. 3(b). From their frequencies, both modes are attributable to lattice modes. The details of the fitting analyses and the assignments of those oscillations are reported in Supplemental Material S4 [40].

The minus-cosine-type oscillation with $56 \mathrm{~cm}^{-1}$ was observed in the reflectivity changes in the previous NIRpump visible-probe spectroscopy and attributed to the lattice mode corresponding to the release of dimerization in photogenerated neutral domains [34], which is generated by the mechanism of displacive excitation of coherent phonon (DECP) [45]. The observation of the same oscillation supports our interpretation that neutral domains are generated by the MIR pulse excitation. This interpretation is not inconsistent with the fact that the similar mode corresponding to the dimeric molecular displacements becomes Raman inactive above $T_{\mathrm{c}}$ [29] because the original neutral phase has the inversion symmetry. On the other hand, the $89 \mathrm{~cm}^{-1}$ oscillation is of a minus sine type. Therefore, this oscillation cannot be attributed to the DECP mechanism but should be ascribed to the oscillation in the original ionic states. A possible mechanism for the generation of this oscillation is a nonlinear coupling 
$[12,46]$ of the IR-active intramolecular vibration $\left(a_{g}\right.$ mode) with the $89 \mathrm{~cm}^{-1}$ mode.

The previous steady-state vibrational spectroscopy [47-49] suggested that IR-active and Raman-active modes associated with the dimerization are located at $\sim 90 \mathrm{~cm}^{-1}$ at $50 \mathrm{~K}$ and show a softening with increase of temperature to $T_{\mathrm{c}}$ in the ionic phase. This result is consistent with our interpretations that the $89 \mathrm{~cm}^{-1}$ oscillation we observed corresponds to the dimerization modes in the ionic phase. For the details of our interpretations, see Supplemental Material S4 [40].

It is important to investigate how TTF-CA responds to an MIR pulse that is not resonant with the $a_{g}$ modes. In our system, we cannot change the frequency of the CEP-stable MIR pulses. Alternatively, using a conventional two-color pump-probe system, we compared the reflectivity changes for the intramolecular transition by MIR pulses that are and are not resonant with $a_{g}$ modes. The results showed that the IN conversion is less efficient with off-resonant excitation than it is with resonant excitation (Supplemental Material S5 [40]). This difference demonstrates that charge modulation within each dimer mediated by EMV coupling is responsible for the IN conversion.

In summary, we demonstrated that a resonant excitation of intramolecular vibration effectively induces the IN conversion in TTF-CA. The MIR-pump subcycle-reflectivity-probe and SHG-probe measurements revealed that the large charge modulations in $D A$ dimers caused by EMV coupling induce the quantum mixing of the neutral excited state with the ionic ground state and result in the conversion to the neutral state, which is accompanied by the coherent molecular oscillation corresponding to the release of dimeric molecular displacements within the neutral state. The same excitation of intramolecular vibration gives rise to another coherent molecular oscillation associated with the dimerization in the original ionic state via the nonlinear coupling. The results presented here show that the resonant excitation of intramolecular vibrations with EMV couplings is an effective way to achieve efficient electronic-state controls in molecular materials.

This Letter was partly supported by Grants-in-Aid for Scientific Research from the Japan Society for the Promotion of Science (JSPS) (Project Nos. JP25247049, JP18H01166, JP18K13476, JP20K03801, and JP21H04988) and by Core Research for Evolutional Science and Technology (CREST) (Grant No. JPMJCR1661), Japan Science and Technology Agency. T. Morimoto and T. Otaki were supported by the JSPS through the Program for Leading Graduate Schools (MERIT). T. Morimoto was supported by JSPS Research Fellowships for Young Scientists.
[1] For a review, Photoinduced phase transitions, edited by K. Nasu (World Scientific, Singapore, 2004).

[2] T. Kampfrath, A. Sell, G. Klatt, A. Pashkin, S. Mährlein, T. Dekorsy, M. Wolf, M. Fiebig, A. Leitenstorfer, and R. Huber, Coherent terahertz control of antiferromagnetic spin waves, Nat. Photon. 5, 31 (2011).

[3] T. Kubacka, J. A. Johnson, M. C. Hoffmann, C. Vicario, S. de Jong, P. Beaud, S. Grübel, S.-W. Huang, L. Huber, L. Patthey, Y.-D. Chuang, J. J. Turner, G. L. Dakovski, W.-S. Lee, M. P. Minitti, W. Schlotter, R. G. Moore, C. P. Hauri, S. M. Koohpayeh, V. Scagnoli et al., Large-amplitude spin dynamics driven by a $\mathrm{THz}$ pulse in resonance with an electromagnon, Science 343, 1333 (2014).

[4] S. Baierl, M. Hohenleutner, T. Kampfrath, A. K. Zvezdin, A. V. Kimel, R. Huber, and R. V. Mikhaylovskiy, Nonlinear spin control by terahertz-driven anisotropy fields, Nat. Photon. 10, 715 (2016).

[5] T. F. Nova, A. Cartella, A. Cantaluppi, M. Först, D. Bossini, R. V. Mikhaylovskiy, A. V. Kimel, R. Merlin, and A. Cavalleri, An effective magnetic field from optically driven phonons, Nat. Phys. 13, 132 (2017).

[6] S. F. Maehrlein, I. Radu, P. Maldonado, A. Paarmann, M. Gensch, A. M. Kalashnikova, R. V. Pisarev, M. Wolf, P. M. Oppeneer, J. Barker, and T. Kampfrath, Dissecting spin-phonon equilibration in ferrimagnetic insulators by ultrafast lattice excitation, Sci. Adv. 4, eear5164 (2018).

[7] D. Fausti, R. I. Tobey, N. Dean, S. Kaiser, A. Dienst, M. C. Hoffmann, S. Pyon, T. Takayama, H. Takagi, and A. Cavalleri, Light-induced superconductivity in a stripe-ordered cuprate, Science 331, 189 (2011).

[8] M. Mitrano, A. Cantaluppi, D. Nicoletti, S. Kaiser, A. Perucchi, S. Lupi, P. Di Pietro, D. Pontiroli, M. Riccò, S. R. Clark,
D. Jaksch, and A. Cavalleri, Possible light-induced superconductivity in $\mathrm{K}_{3} \mathrm{C}_{60}$ at high temperature, Nature (London) 530, 461 (2016).

[9] M. Bussi, D. Nicoletti, M. Fechner, N. Tancogne-Dejean, M. A. Sentef, A. Georges, T. Biesner, E. Uykur, M. Dressel, A. Henderson, T. Siegrist, J. A. Schlueter, K. Miyagawa, K. Kanoda, M.-S. Nam, A. Ardavan, J. Coulthard, J. Tindall, F. Schlawin, D. Jaksch et al., Photomolecular HighTemperature Superconductivity, Phys. Rev. X 10, 031028 (2020).

[10] R. Matsunaga, N. Tsuji, H. Fujita, A. Sugioka, K. Makise, Y. Uzawa, H. Terai, Z. Wang, H. Aoki, and R. Shimano, Lightinduced collective pseudospin precession resonating with Higgs mode in a superconductor, Science 345, 1145 (2014).

[11] M. Rini, R. Tobey, N. Dean, J. Itatani, Y. Tomioka, Y. Tokura, R. W. Schoenlein, and A. Cavalleri, Control of the electronic phase of a manganite by mode-selective vibrational excitation, Nature (London) 449, 72 (2007).

[12] R. Mankowsky, A. Von Hoegen, M. Först, and A. Cavalleri, Ultrafast Reversal of the Ferroelectric Polarization, Phys. Rev. Lett. 118, 197601 (2017).

[13] A. von Hoegen, R. Mankowsky, M. Fechner, M. Först, and A. Cavalleri, Probing the interatomic potential of solids with strong-field nonlinear phononics, Nature (London) 555, 79 (2018).

[14] M. Kozina, M. Fechner, P. Marsik, T. van Driel1, J. M. Glownia, C. Bernhard, M. Radovic, D. Zhu, S. Bonetti, U. Staub, and M. C. Hoffmann, Terahertz-driven phonon upconversion in $\mathrm{SrTiO}_{3}$, Nat. Phys. 15, 387 (2019).

[15] T. Umanodan, K. Kaneshima, K. Takeuchi, N. Ishii, J. Itatani, H. Hirori, Y. Sanari, K. Tanaka, Y. Kanemitsu, T. Ishikawa, S. Koshihara, S. Horiuchi, and Y. Okimoto, Ultrafast control 
of ferroelectricity with dynamical repositioning of protons in a supramolecular cocrystal studied by femtosecond nonlinear spectroscopy, J. Phys. Soc. Jpn. 88, 013705 (2019).

[16] X. Li, T. Qiu, J. Zhang, E. Baldini, J. Lu, A. M. Rappe, and K. A. Nelson, Terahertz field-induced ferroelectricity in quantum paraelectric $\mathrm{SrTiO}_{3}$, Science 364, 1079 (2019).

[17] S. Kaiser, S. R. Clark, D. Nicoletti, G. Cotugno, R. I. Tobey, N. Dean, S. Lupi, H. Okamoto, T. Hasegawa, D. Jaksch, and A. Cavalleri, Optical properties of a vibrationally modulated solid state Mott insulator, Sci. Rep. 4, 3823 (2014).

[18] R. Singla, G. Cotugno, S. Kaiser, M. Forst, M. Mitrano, H. Y. Liu, A. Cartella, C. Manzoni, H. Okamoto, T. Hasegawa, S. R. Clark, D. Jaksch, and A. Cavalleri, THz-Frequency Modulation of the Hubbard $U$ in an Organic Mott Insulator, Phys. Rev. Lett. 115, 187401 (2015).

[19] M. J. Rice, Organic Linear Conductors as Systems for the Study of Electron-Phonon Interactions in the Organic Solid State, Phys. Rev. Lett. 37, 36 (1976).

[20] A. Girlando, R. Bozio, C. Pecile, and J. B. Torrance, Discovery of vibronic effects in the Raman spectra of mixed-stack chargetransfer crystals, Phys. Rev. B 26, 2306(R) (1982).

[21] J. B. Torrance, J. E. Vazquez, J. J. Mayerle, and V. Y. Lee, Discovery of a Neutral-to-Ionic Phase Transition in Organic Materials, Phys. Rev. Lett. 46, 253 (1981).

[22] J. B. Torrance, A. Girlando, J. Mayerle, J. I. Crowley, V. Y. Lee, P. Batail, and S. J. Laplaca, Anomalous Nature of Neutralto-Ionic Phase Transition in Tetrathiafulvalene-Chloranil, Phys. Rev. Lett. 47, 1747 (1981).

[23] M. Le Cointe, M. H. Lemée-Cailleau, H. Cailleau, B. Toudic, L. Toupet, G. Heger, F. Moussa, P. Schweiss, K. H. Kraft, and N. Karl, Symmetry breaking and structural changes at the neutralto-ionic transition in tetrathiafulvalene- $p$-chloranil, Phys. Rev. B 51, 3374 (1995).

[24] S. Horiuchi, Y. Okimoto, R. Kumai, and Y. Tokura, Anomalous Valence Fluctuation Near a Ferroelectric Transition in an Organic Charge-Transfer Complex, J. Phys. Soc. Jpn. 69, 1302 (2000).

[25] N. Nagaosa, Theory of neutral-ionic transition in organic crystals. III. Effect of the electron-lattice interaction, J. Phys. Soc. Jpn. 55, 2754 (1986).

[26] K. Kobayashi, S. Horiuchi, R. Kumai, F. Kagawa, Y. Murakami, and Y. Tokura, Electronic Ferroelectricity in a Molecular Crystal with Large Polarization Directing Antiparallel to Ionic Displacement, Phys. Rev. Lett. 108, 237601 (2012).

[27] S. Ishibashi and K. Terakura, Exotic ferroelectricity in tetrathiafulvalene- $p$-chloranil: anomalous effective charges and a picture in the framework of maximally localized Wannier orbitals, J. Phys. Soc. Jpn. 83, 073702 (2014).

[28] K. Terakura and S. Ishibashi, Mechanism of covalency-induced electric polarization within the framework of maximally localized Wannier orbitals, Phys. Rev. B 91, 195120 (2015).

[29] T. Miyamoto, H. Yada, H. Yamakawa, and H. Okamoto, Ultrafast modulation of polarization amplitude by terahertz fields in electronic-type organic ferroelectrics, Nat. Commun. 4, 2586 (2013).

[30] T. Morimoto, T. Miyamoto, and H. Okamoto, Ultrafast electron and molecular dynamics in photoinduced and electric-fieldinduced neutral-ionic transitions, Crystals 7, 132 (2017).

[31] A. Painelli and A. Girlando, Electron-molecular vibration (e-mv) coupling in charge-transfer compounds and its con- sequences on the optical spectra: a theoretical framework, J. Chem. Phys. 84, 5655 (1986).

[32] M. Dressel and T. Peterseim, Infrared investigations of the neutral-ionic phase transition in TTF-CA and its dynamics, Crystals 7, 17 (2017).

[33] S. Koshihara, Y. Tokura, T. Mitani, G. Saito, and T. Koda, Photoinduced valence instability in the organic molecular compound tetrathiafulvalene- $p$-chloranil (TTF-CA), Phys. Rev. B 42, 6853(R) (1990).

[34] H. Okamoto, Y. Ishige, S. Tanaka, H. Kishida, S. Iwai, and Y. Tokura, Photoinduced phase transition in tetrathiafulvalenep-chloranil observed in femtosecond reflection spectroscopy, Phys. Rev. B 70, 165202 (2004).

[35] H. Uemura and H. Okamoto, Direct Detection of the Ultrafast Response of Charges and Molecules in the Photoinduced Neutral-to-Ionic Transition of the Organic Tetrathiafulvalenep-Chloranil Solid, Phys. Rev. Lett. 105, 258302 (2010).

[36] T. Morimoto, T. Miyamoto, H. Yamakawa, T. Terashige, T. Ono, N. Kida, and H. Okamoto, Terahertz-Field-Induced Large Macroscopic Polarization and Domain-Wall Dynamics in an Organic Molecular Dielectric, Phys. Rev. Lett. 118, 107602 (2017).

[37] T. Morimoto, N. Sono, T. Miyamoto, N. Kida, and H. Okamoto, Generation of a carrier-envelope-phase-stable femtosecond pulse at $10 \mu \mathrm{m}$ by direct down-conversion from a Ti:sapphire laser pulse, Appl. Phys. Express 10, 122701 (2017).

[38] A. Shirakawa, I. Sakane, M. Takasaka, and T. Kobayashi, Sub-5-fs visible pulse generation by pulse-front-matched noncollinear optical parametric amplification, Appl. Phys. Lett. 74, 2268 (1999).

[39] S. Adachi, Y. Watanabe, Y. Sudo, and T. Suzuki, Few-cycle pulse generation from noncollinear optical parametric amplifier with static dispersion compensation, Chem. Phys. Lett. 683, 7 (2017).

[40] See Supplemental Material at http://link.aps.org/supplemental/ 10.1103/PhysRevResearch.3.L042028, which includes experimental details with related Refs. [41,42], the estimation of modulation magnitude of degree of CT by the MIR pulse, the fitting analyses of SHG changes by the MIR pulse, and the pump-frequency dependence of reflectivity changes.

[41] T. Miyamoto, Y. Matsui, T. Terashige, T. Morimoto, N. Sono, H. Yada, S. Ishihara, Y. Watanabe, S. Adachi, T. Ito, K. Oka, A. Sawa, and H. Okamoto, Probing ultrafast spin-relaxation and precession dynamics in a cuprate Mott insulator with seven-femtosecond optical pulses, Nat. Commun. 9, 3948 (2018).

[42] R. Trebino, K. W. Delong, D. N. Fittinghoff, J. N. Sweetser, M. A. Krumbügel, B. A. Richman, and D. J. Kane, Measuring ultrashort laser pulses in the time-frequency domain using frequency-resolved optical gating, Rev. Sci. Instrum. 68, 3277 (1997).

[43] T. Luty, H. Cailleau, S. Koshihara, E. Collet, M. Takesada, M. H. Lemée-Cailleau, M. Buron-Le Cointe, N. Nagaosa, Y. Tokura, E. Zienkiewicz, and B. Ouladdiaf, Static and dynamic order of cooperative multi-electron transfer, Europhys. Lett. 59, 619 (2002).

[44] H. Yamakawa, T. Miyamoto, T. Morimoto, H. Yada, Y. Kinoshita, M. Sotome, N. Kida, K. Yamamoto, K. Iwano, 
Y. Matsumoto, S. Watanabe, Y. Shimoi, M. Suda, H. M. Yamamoto, H. Mori, and H. Okamoto, Novel electronic ferroelectricity in an organic charge-order insulator investigated with terahertz-pump optical-probe spectroscopy, Sci. Rep. 6, 20571 (2016).

[45] H. J. Zeiger, J. Vidal, T. K. Cheng, E. P. Ippen, G. Dresselhaus, and M. S. Dresselhaus, Theory for displacive excitation of coherent phonons, Phys. Rev. B 45, 768 (1992).

[46] R. Mankowsky, M. Först, and A. Cavalleri, Non-equilibrium control of complex solids by nonlinear phononics, Rep. Prog. Phys. 79, 064503 (2016).
[47] A. Moreac, A. Girard, Y. Delugeard, and Y. Marqueton, The neutral-to-ionic phase transition of TTF-CA: a Raman and infrared study versus temperature at atmospheric pressure, J. Phys. Condens. Matter 8, 3553 (1996).

[48] M. Masino, A. Girlando, A. Brillante, R. G. Della Valle, E. Venuti, N. Drichko, and M. Dressel, Lattice dynamics of TTFCA across the neutral-ionic transition, Chem. Phys. 325, 71 (2006).

[49] M. Masino, N. Castagnetti, and A. Girlando, Phenomenology of the neutral-ionic valence instability in mixed stack chargetransfer crystals, Crystals 7, 108 (2017). 